\title{
Circadian Period and the Melatonin Rhythm in Men and Women: Predictors of Sleep during the Weekend and in the Laboratory
}

\section{Running title: Circadian period and weekend sleep}

$$
\text { JOSR-12-134-R1 }
$$

\footnotetext{
Alpar S Lazar*1, Nayantara Santhi*1, Sibah Hasan*¹, June Chi-Yan Lo ${ }^{1}$, Jonathan D Johnston $^{1,2}$, Malcolm von Schantz ${ }^{1,2}$, Simon N Archer ${ }^{1,2}$, Derk-Jan Dijk ${ }^{1,2}$

1 Surrey Sleep Research Centre, Faculty of Health and Medical Sciences, University of Surrey GU2 7XP United Kingdom,
}

2 Department of Biochemical Sciences and Physiology, Faculty of Health and Medical Sciences, University of Surrey, Guildford, Surrey GU2 7XH United Kingdom

\section{* These authors contributed equally to this work.}

\section{Words:}

Abstract: 250

Main text: 2135

Figures: 3

Tables: 1

References: 16

Key words: diurnal preference, sleep disorders, forced desynchrony, social jet leg, insomnia, delayed sleep phase syndrome

\section{Corresponding author}

Alpar S Lazar, PhD

Surrey Sleep Research Centre, Faculty of Health and Medical Sciences, University of Surrey, Egerton Road, Guildford, Surrey GU2 7XP, UK email:a.lazar@surrey.ac.uk Telephone + 44 (0)788 3271351 


\section{Conflict of interest:}

This is not an industry sponsored study. Prof Dijk has received research funding from, and served as a consultant to, Philips Lighting and several pharmaceutical companies with an interest in sleep and circadian rhythms. He is also Editor in Chief of JSR but he has had no involvement in the processing of this manuscript. Drs Lazar, Santhi, Hasan, Lo, Johnston, Archer, and von Schantz declare no conflicts of interest. 


\section{Summary}

Sleep complaints and irregular sleep patterns, such as curtailed sleep during workdays and longer and later sleep during weekends are common. It is often implied that differences in circadian period and in entrained phase contribute to these patterns but few data are available. We assessed parameters of the circadian rhythm of melatonin at baseline and in a forced desynchrony protocol in 35 participants (18 women) with no sleep disorders. Circadian period varied between $23 \mathrm{~h} 50 \mathrm{~min}$ and $24 \mathrm{~h} 31 \mathrm{~min}$ and correlated positively $(n=31$, $\mathrm{r}_{\mathrm{s}}=0.43, P=0.017$ ) with the timing of the melatonin rhythm relative to habitual bedtime. This phase of the melatonin rhythm correlated with the insomnia severity index $\left(n=35, \mathrm{r}_{\mathrm{s}}=0.47\right.$, $P=0.004)$. Self-reported time in bed (TIB) during free days also correlated with the timing of the melatonin rhythm $\left(n=35, \mathrm{r}_{\mathrm{s}}=0.43, P=0.01\right)$ as well as with circadian period $\left(n=31, \mathrm{r}_{\mathrm{s}}=0.47\right.$, $P=0.007)$ such that individuals with a more delayed melatonin rhythm or a longer circadian period reported longer sleep during the weekend. The increase in TIB during the free days correlated positively with circadian period $\left(n=31, \mathrm{r}_{\mathrm{s}}=0.54, P=0.002\right)$. Polysomnographicallyassessed latency to persistent sleep $\left(n=34, \mathrm{r}_{\mathrm{s}}=0.48, P=0.004\right)$ correlated with the timing of the melatonin rhythm when participants were sleeping at their habitual bedtimes in the laboratory. This correlation was significantly stronger in women than in men $(\mathrm{Z}=2.38, P=0.017)$. The findings show that individual differences in circadian period and phase of the melatonin rhythm associate with differences in sleep and suggest that individuals with a long circadian period may be at risk of developing sleep problems. 


\section{Introduction}

Irregular sleep patterns, delayed sleep phase syndrome, non-24-h sleep-wake syndrome and insomnia complaints are prevalent in modern societies (Sack et al., 2007). This may be in part because, with the advent of artificial light, sleep-wake timing is no longer dictated by the natural 24-h light-dark cycle. The exposure to artificial light associated with work schedules and evening behaviours such as watching TV or working in front of computer screens, has been shown to affect circadian physiology and latency to sleep onset (Santhi et al., 2011; Cajochen et al. 2011) and appears to be a major driver of sleep timing. Our weekly work schedules often lead to a pattern of irregular sleep with delayed and short sleep during workdays and sleep rebound during weekends/free days (Basner et al., 2007.) To our knowledge, biological correlates of these sleep patterns and individual differences therein have not been identified. The involvement of circadian processes is often implied although the available evidence primarily consist of the association between self-reported circadian preference and self reported delayed sleep during free days (Roenneberg et al., 2003).

Circadian rhythms with their near-24-h period are generated by a network of transcriptional activators and repressors and synchronised to the 24-h day by light (Takahashi et al., 2008). Laboratory studies have established that sleep timing and sleep quality are regulated by a fine-tuned interaction of sleep homeostasis and circadian rhythmicity (Dijk and Czeisler 1995).

Based on our current understanding of the light-sensitivity and photic- entrainment of circadian clocks, and the homeostatic and circadian interaction in the regulation of sleep, it can be predicted that individuals with a long circadian period are at risk for delayed and irregular sleep patterns (Dijk and Lockley, 2002). This because self-selected artificial light exposure in the evening will lead to delay of the onset of melatonin, sleepiness and latency to sleep onset (Santhi et al., 2011), and hence exacerbate the natural tendency to delay, especially, in individuals with a long circadian period. While during workdays this delay cannot be reflected in wake time, during weekends it can lead to later sleep times, and create irregular sleep patterns.

However, no direct evidence supporting this prediction is currently available. We assessed associations between the circadian period and entrained phase of the melatonin rhythm and also compared men and women because gender differences in entrained phase (Cain et al., 2010) and circadian period (Duffy et al., 2011) have been reported. 


\section{Methods}

The protocol received a favourable opinion from the Research Ethics Committee of the University of Surrey. All 35 healthy non-smoking participants (18 females) aged 20.5-32.4 provided written informed consent and, after passing a screening night, entered a forced desynchrony protocol. Details of the recruitment, screening procedures and protocol have been reported elsewhere (Hasan et al., 2012). This sample was stratified on the basis of the rs57875989 PER3 polymorphism and in vivo and in vitro circadian period were compared across genotypes, but no differences between genotypes were observed (Hasan et al., 2012) and genotype will not be considered in the current analyses.

During a screening visit, participants completed multiple questionnaires. Self-reported sleep habits during workdays and free days were assessed with the Munich Chronotype Questionnaire (MCTQ) (Roenneberg et al., 2003). The MCTQ assesses prevailing sleep habits and addresses sleep-wake timing separately for workdays and rest days. We extracted bedtime, wakeup time as well as sleep latency from the MCTQ. From these measures we derived reported time in bed (TIB) as the time period between reported bedtime and wakeup time. Sleep complaints were assessed with the Insomnia Severity Index (ISI). The ISI is a 7item questionnaire assessing dissatisfaction with sleep during the last 2 weeks, with a maximum score of 28. A score of 0-7 indicates no clinically significant insomnia and a score of 8-14 is considered sub-threshold insomnia (Bastien et al., 2001). Sleep disorders were excluded by a clinical polysomnographic assessment during the screening night.

In the week prior to the laboratory phase, participants were instructed to follow a regular sleep-wake schedule and compliance was assessed by actigraphy. During the 9-day laboratory protocol, participants were resident in the Clinical Research Centre of the University of Surrey. After a baseline day, participants were scheduled to live on 28-h 'days' consisting of 9h20 min in bed in darkness and 18h40min wakefulness in dim light (<5 lx) in the absence of external time cues (Figure 1). This leads to desynchrony between the sleep-wake cycle and circadian rhythms and allows the assessment of the near-24-h circadian period of the hypothalamic circadian pacemaker driving circadian rhythms such as plasma melatonin when the desynchrony is at least one beat cycle long as in the current protocol (Dijk and Czeisler 1995; Klerman et al., 1996). During the sleep periods of the $1^{\text {st }}, 4^{\text {th }}$ and $7^{\text {th }}$ sleep/wake cycle of the forced desynchrony protocol, blood samples were scheduled to be collected every 60 or 120 minutes for plasma melatonin analysis. The timing of the onset of 
nocturnal melatonin secretion while participants were in dim light (the dim light melatonin onset or DLMO, defined as the time when the melatonin concentration was $25 \%$ of the measured peak value) was used as a circadian phase marker (see Hasan et al 2012 for rationale and reference). The timing of melatonin onset at baseline (the evening prior to sleep period 2) was assessed in 35 participants and intrinsic circadian period was determined from the progression of the melatonin rhythm throughout the protocol in 31 participants, as described in Hasan et al., 2012. The data on circadian period, melatonin phase and diurnal preference as reported in Hasan et al. 2012, were used as predictor variables in the current analyses.

Throughout the laboratory segment, sleep was recorded polysomnographically on Siesta 802 devices (Compumedics, Abbotsford, VIC, Australia) using a 12-channel EEG montage. Sleep staging was performed according to the criteria of Rechtschaffen and Kales and standard sleep parameters were computed. In addition we computed Latency To Persistent Sleep, a parameter often used in clinical trials in insomnia and defined as the first occurrence of any sleep stage that is followed by 10 minutes of uninterrupted sleep.

Statistical analyses were based on Spearman correlations, multiple regression analyses and analyses of covariance as implemented in SAS version 9.1 (SAS Institute, Cary, NC). Correlation coefficients were Fisher-z transformed and statistically compared between the two gender groups using a T-test.

\section{Results}

Circadian period varied among individuals between 23h50min and 24h31min with an average of $24 \mathrm{~h} 10 \mathrm{~min}$ (S.D.=10min). This variation correlated significantly $\left(n=31, \mathrm{r}_{\mathrm{s}}=0.43, P=0.017\right)$ with entrained phase of the melatonin rhythm such that, in individuals with a longer period, habitual bedtime (i.e. dark onset) occurred closer to the onset of melatonin secretion (Hasan et al., 2012). There was no statistically significant difference between the genders for either circadian period or the timing of the onset of melatonin secretion relative to habitual bedtime (Table 1).

The average insomnia severity index was 3.7 (S.D.=3.5, $n=35$ ), varied between individuals from 0 to 12 , and correlated with circadian period $\left(n=31, \mathrm{r}_{\mathrm{s}}=0.36, P=0.05\right)$. The insomnia severity index also correlated significantly $\left(n=35, \mathrm{r}_{\mathrm{s}}=0.47, P=0.004\right)$ with a more delayed timing of the melatonin rhythm at baseline (Figure 2A). 
Average bed and wakeup times during free days were significantly later than during workdays [bed time: 23h34min (S.D.=1h14 min) vs. 00h28min (S.D.=1h17min), $n=35$, T=6.365, $P<0.001$; wake time: 07h45min (S.D.=1h13min), vs. 09h13min (S.D.=1h22min), $n=35, \mathrm{~T}=6.198, P<0.001$ ]. TIB during workdays averaged 8h10min (S.D.=1h1min), whereas, during free days, it increased to 8 h46min (S.D.=1h6min) $(n=35, \mathrm{~T}=-2.722, P<0.01)$. An increase in TIB or no change was observed in the majority of participants, although in some participants a decrease was observed (Figure 2B). On average females showed a 61 minutes earlier bedtime $\left(F_{1.32}=6.44, P=0.016\right)$ and 59 minutes earlier wakeup time $\left(F_{1.32}=5.74\right.$, $P=0.023$ ) during free days as compared to males. However, no significant effect of gender was observed for sleep timing during workdays or any of the TIB measures.

Circadian period correlated with 'weekend' sleep timing parameters such that participants with a longer circadian period reported longer TIB during free days $\left(n=31, \mathrm{r}_{\mathrm{s}}=0.47, P=0.007\right)$ and a greater increase in TIB from workdays to free days ( $\left.n=31, \mathrm{r}_{\mathrm{s}}=0.54, P=0.002\right)$ (Figure 2B). Multivariate regression analyses showed that TIB during free days was independently and positively predicted by both circadian period $(P=0.009)$ and TIB during workdays $(P=0.02)$. The change in TIB from workdays to free days was also independently and positively predicted by circadian period $(P=0.009)$ and negatively by TIB during workdays $(P=0.007)$. Sleep-wake timing parameters during workdays were not associated with circadian period $(P>0.4$ in all cases).

A more delayed melatonin rhythm was associated with a longer sleep latency $\left(n=34, \mathrm{r}_{\mathrm{s}}=0.39\right.$, $P=0.024)$ as well as a longer latency to persistent sleep $\left(n=34, \mathrm{r}_{\mathrm{s}}=0.48, P=0.004\right)$, as assessed polysomnographically when participants were sleeping at their habitual bedtime. These correlations were significantly stronger in women than in men for both sleep latency $(Z=-$ 2.02, $P=0.043)$ and latency to persistent sleep $(\mathrm{Z}=2.38, P=0.017)$ (Figure 3). Other baseline sleep parameters were not associated with either circadian period or phase at baseline and no additional gender differences were observed (Table 1).

\section{Discussion}

The data show that the circadian period of the melatonin rhythm and the timing of the melatonin rhythm relative to habitual bedtime correlated with TIB during free days, the increase in TIB from workdays to free days and the subjective sleep complaints as measured by the insomnia severity index, as well as polysomnographically-assessed sleep latency when 
participants were sleeping at their habitual sleep time. The direction of these correlations is such that individuals with a long circadian period and delayed melatonin rhythm appear most at risk of delayed sleep during weekends, and experience less sleep satisfaction in general. The observation that sleep timing during workdays did not correlate with circadian period or the entrained phase of the melatonin rhythm confirms that, in contrast to free days, sleepwake timing during workdays is primarily determined by social demands such as work schedules and social activities.

The most parsimonious interpretation of these observations is that while living in an environment with access to artificial light, a long circadian period is associated with a delay of the melatonin rhythm relative to bedtime (Wright et al., 2005). During workdays, when sleep timing is primarily determined by social factors, sleep occurs at a sub-optimal phase relative to the sleep propensity and melatonin rhythm and this leads to sleep complaints. It is only during the weekend that sleep occurs in phase with the sleep propensity rhythm, which in individuals with a long circadian period occurs later than in those with a shorter circadian period. The accumulated sleep debt carried over from workdays is greater in individuals with longer intrinsic circadian period, who thus show a greater increase in sleep duration during free days. This interpretation is supported by the multiple regression analyses, which showed that the increase in TIB during free days is correlated positively with circadian period and negatively with TIB during workdays. This implies that the increase in TIB during free days reflects both the timing of the circadian sleep propensity rhythm and a homeostatic response to short sleep during workdays. Based on subjective reports it appeared that some participants had shorter sleep on weekends than on weekdays. This may be related to late social activities on weekends, or reflect inaccuracies in the subjective data. More objective long-term data on sleep timing during workdays and free days are required to fully understand the dynamics of sleep duration in the real world.

Although in this small sample $(n=31)$ with limited statistical power we did not replicate the previously reported gender differences in entrained phase (Cain et al., 2010) (n=57) and circadian period (Duffy et al., 2011), (n=157), we did, however, confirm gender differences in sleep timing. The observed gender differences in the strength of the correlation between circadian parameters and sleep physiology add to the growing body of evidence of sex differences in circadian rhythmicity (Mong et al., 2011). 
The current sample was stratified with respect to the PER3 VNTR polymorphism and it can be argued that this may have influenced some of the results also because the PER3 $3^{4 / 4}$ genotype has been associated with insomnia severity in alcohol dependence (Brower et al., 2012). However, there were no significant genotype-dependent differences in circadian period, entrained phase or habitual sleep-wake timing. Nevertheless, this study population, as well as other study populations in this research area was highly selected, and how these results will generalise to a more representative study population remains to be investigated.

Overall, our data show that intrinsic characteristics of the circadian timing system assessed in the absence of the confounding influence of work, sleep and light exposure schedules, associate with sleep characteristics while during the weekend and while sleeping at habitual bedtime in the laboratory. The data imply that individuals with a long circadian period may be more at risk of developing irregular sleep wake patterns, sleep complaints and a delayed sleep phase syndrome.

\section{Acknowledgements}

Funded by BBSRC grant (BB/F022883/1). We thank the staff of the Clinical Research Centre for their help in data collection, and Patrick McCabe for statistical advice. 


\section{References}

Basner, M., Fomberstein, K.M., Razavi, F.M. et al. American time use survey: sleep time and its relationship to waking activities. Sleep, 2007, 30: 1085-95.

Bastien, C.H., Vallières, A. and Morin, C.M. Validation of the Insomnia Severity Index as an outcome measure for insomnia research. Sleep Med., 2001, 2: 297-307.

Brower, K.J., Wojnar, M., Sliwerska, E., Armitage, R. and Burmeister, M. PER3 polymorphism and insomnia severity in alcohol dependence. Sleep, 2012, 35: 571-7.

Cain, S.W., Dennison, C.F., Zeitzer, J.M. et al. Sex differences in phase angle of entrainment and melatonin amplitude in humans. J. Biol. Rhythms, 2010, 25: 288-96.

Cajochen, C., Frey, S., Anders, D. et al. Evening exposure to a light-emitting diodes (LED)backlit computer screen affects circadian physiology and cognitive performance. $J$. Appl. Physiol., 2011, 110: 1432-8.

Dijk, D.J. and Czeisler, C.A. Contribution of the circadian pacemaker and the sleep homeostat to sleep propensity, sleep structure, electroencephalographic slow waves, and sleep spindle activity in humans. J. Neurosci., 1995, 15: 3526-38.

Dijk, D.J. and Lockley, S.W. Integration of human sleep-wake regulation and circadian rhythmicity. J. Appl. Physiol., 2002, 92: 852-62.

Duffy, J.F., Cain, S.W., Chang, A.M. et al., Sex difference in the near-24-hour intrinsic period of the human circadian timing system. Proc. Natl. Acad. Sci. U. S. A., 2011, 108: $15602-8$.

Hasan, S., Santhi, N., Lazar, A.S. et al. Assessment of circadian rhythms in humans: comparison of real-time fibroblast reporter imaging with plasma melatonin. FASEB $J$. 2012, 26: 2414-2423.

Klerman, E.B., Dijk, D.J., Kronauer, R.E. et al. Simulations of light effects on the human_circadian_pacemaker: implications for assessment of intrinsic period. Am. J. Physiol., 1996, 270: R271-82.

Mong, J.A., Baker, F.C., Mahoney, M.M., et al. Sleep, rhythms, and the endocrine brain: influence of sex and gonadal hormones. J. Neurosci., 2011, 31: 16107-16.

Roenneberg, T., Wirz-Justice, A. and Merrow, M. Life between clocks: daily temporal patterns of human chronotypes. J. Biol. Rhythms, 2003, 18: 80-90.

Sack, R.L., Auckley, D., Auger, R.R. et al. Circadian rhythm sleep disorders: part II, advanced sleep phase disorder, delayed sleep phase disorder, free-running disorder, and irregular sleep-wake rhythm. An American Academy of Sleep Medicine review. Sleep, 2007, 30: 1484-501. 
Santhi N, Thorne HC, van der Veen DR et al. The spectral composition of evening light and individual differences in the suppression of melatonin and delay of sleep in humans. $J$. Pineal Res., 2011, 53:47-59.

Takahashi, J.S., Shimomura, K. et al. Searching for genes underlying behavior: lessons from circadian rhythms. Science, 2008, 322: 909-12.

Wright, K.P. Jr, Gronfier, C., Duffy, J.F. et al. Intrinsic period and light intensity determine the phase relationship between melatonin and sleep in humans.

J. Biol. Rhythms, 2005, 20: 168-77. 


\section{Legends}

\section{Table 1.}

Circadian and baseline sleep measures in men and women. Number of observations, mean values, standard deviations and one-way analysis effects are indicated.

Sleep Efficiency $=$ TST/TIB; Stage $1(\%)=$ Stage 1 expressed as percentage of TST. Differences between the sexes were assessed with analysis of covariance using age as a covariate.

\section{Figures}

Figure 1.

A doubled-plotted raster plot of the 28-h forced desynchrony protocol (BB0289, 27 years, female). Consecutive sleep periods are plotted both next to (hatched and filled box) and below each other. Melatonin profiles are plotted and the dim light melatonin onset (DLMO) is indicated (red arrow).

Figure 2.

(A) Association of the timing of melatonin onset relative to habitual bedtime ( $n=35$, $\left.\mathrm{r}_{\mathrm{s}}=0.47, P=0.004\right)$ with subjective insomnia complaints as measured by the Insomnia Severity Scale. Negative values on the $\mathrm{X}$ axis indicate that the dim light melatonin onset (DLMO) occurred before habitual bedtime and values close to 0 indicate a more delayed DLMO.

(B) Association of circadian period with the change in Time in bed (TIB) from workdays to free days $\left(n=31, \mathrm{r}_{\mathrm{s}}=0.47, P=0.007\right)$. The values indicate TIB on free days minus TIB on workdays.

Figure 3.

Association of latency to persistent sleep with the timing of melatonin onset relative to bedtime in the entire group $\left(n=34, \mathrm{r}_{\mathrm{s}}=0.48, P=0.004\right)$ and separately for men $\left(n=16, \mathrm{r}_{\mathrm{s}}=0.1\right.$, $=0.723)$ and women $\left(n=18, \mathrm{r}_{\mathrm{s}}=0.76, P<0.001\right)$. Latency to persistent sleep is defined as the time period between lights out and the first 10 minutes of continuous sleep. The difference between the correlation in males and females is significant $(\mathrm{Z}=2.38, P=0.017)$. 